\title{
The VHE SED modelling of Markarian 501 in 2009
}

\author{
S. Sahu, ${ }^{1 \star}$ C. E. López Fortín, ${ }^{1 \star}$ M. E. Iglesias Martínez, ${ }^{2,3 \star}$ S. Nagataki ${ }^{4,5}$ \\ and P. Fernández de Córdoba ${ }^{3}$ \\ ${ }^{1}$ Instituto de Ciencias Nucleares, Universidad Nacional Autónoma de México, Circuito Exterior, C.U., A. Postal 70-543, 04510 Mexico DF, Mexico \\ ${ }^{2}$ Departamento de Telecomunicaciones, Universidad de Pinar del Río, Pinar del Río, Martí \# 270, CP: 20100, Cuba \\ ${ }^{3}$ Instituto Universitario de Matemática Pura y Aplicada, Universitat Politècnica de València (UPV), Camino de Vera s/n, 46022 Valencia, España \\ ${ }^{4}$ Astrophysical big bang Laboratory, RIKEN, Hirosawa, Wako, Saitama 351-0198, Japan \\ ${ }^{5}$ Interdisciplinary Theoretical \& Mathematical Science (iTHEMS), RIKEN, Hirosawa, Wako, Saitama 351-0198, Japan
}

Accepted 2019 December 30. Received 2019 December 6; in original form 2019 September 30

\begin{abstract}
The high energy blazar, Markarian 501 was observed as a part of multi-instrument and multiwavelength campaign spanning the whole electromagnetic spectrum for 4.5 months during March 15 to August 1, 2009. On May 1, Whipple $10 \mathrm{~m}$ telescope observed a very strong $\gamma$-ray flare in a time interval of about $0.5 \mathrm{~h}$. Apart from this flare, high state and low state emissions were also observed by Whipple, VERITAS and MAGIC telescopes. Using the photohadronic model and accounting for the absorption of the extragalactic background light to these very high energy $\gamma$-rays, excellent fits are obtained for the observed spectra. We have shown that the intrinsic spectrum for low state emission is flat, however, for high and very high states this is a power-law with slowly increasing exponent.
\end{abstract}

Key words: astroparticle physics - BL Lacertae objects: general-gamma-rays: galaxies.

\section{INTRODUCTION}

Markarian 501 (Mrk 501) is a high energy peaked blazar (HBL) at a redshift of $z=0.034$ and one of the brightest extragalactic sources in the X-ray/TeV sky. Mrk 501 is identified as a very high energy (VHE, >100 GeV) emitter by the Whipple telescope in 1996 (Abdo et al. 2009). Since its discovery, it has been the subject of extensive studies in multiwavelengths as it has undergone many major outbursts on long time scales and rapid flares on short time scales mostly in the X-rays and TeV energies (Ghisellini et al. 1998; Aharonian et al. 1999; Aharonian 1999, 2001; Pian et al. 1999; Villata \& Raiteri 1999; Sambruna et al. 2000; Katarzyński, Sol \& Kus 2001; Tavecchio et al. 2001; Krawczynski et al. 2004; Gliozzi et al. 2006). As a part of multiwavelength campaign covering a period of 4.5 months from March 15 to August 1, 2009, Mrk 501 was observed by multi-instruments ( $~ 30$ different instruments) covering the entire electromagnetic spectrum (Aliu et al. 2016; Ahnen et al. 2017). In the optical and radio bands the flux was found to be almost constant and in the UV band it had some variation, however, during the VHE flare the flux was constant. Around the epoch of VHE flaring only, the X-ray light curve exhibited variation. In the $\gamma$-ray range from $0.1 \mathrm{GeV}$ to $20 \mathrm{TeV}$, it was observed by Fermi-LAT, MAGIC, VERITAS and Whipple $10 \mathrm{~m}$ telescopes. The $\gamma$-ray telescopes observed two episodes of flaring, one on 1st May

\footnotetext{
^E-mail: sarira@nucleares.unam.mx (SS); carlos.fortin@correo.nucleares. unam.mx (CELF); shigehiro.nagataki@riken.jp (MEIM)
}

(MJD 54952) and another on 22nd May (MJD 54973). In the VHE domain, statistically significant variation in the flux was observed by all the instruments.

The VHE flare of May 1, 2009 was first observed by the Whipple $10 \mathrm{~m}$ telescope when a high emission state was detected above 300 $\mathrm{GeV}$ (Aliu et al. 2016; Ahnen et al. 2017). A sudden increase in the flux in the first $0.5 \mathrm{~h}$ (MJD 54952.31 to MJD 54952.37) by about one order of magnitude compared to the average flux measured during the full campaign was recorded (Aliu et al. 2016). Following an alert by the Whipple telescope, $1.4 \mathrm{~h}$ later (MJD 54952.41), VERITAS continued simultaneous observation with the Whipple and detected elevated level of flux without statistically significant variation in it during the full period of observation (MJD 54952.41 to MJD 54952.48). Both Whipple and VERITAS observed elevated flux level in the source until MJD 54955.

The MAGIC collaboration participated in this campaign with a single telescope only. Also due to upgrading of the telescope, data were not taken during the whole campaign period. However, on May 22 (MJD 54973), the MAGIC telescope observed the blazar for $1.7 \mathrm{~h}$ and an elevated VHE flux (a VHE flare) $\sim 3$ times the low flux level has been recorded (Ahnen et al. 2017). During this flaring period, no significant intra-night variability was observed. The VHE $\gamma$-ray spectra observed during the 4.5 months period by different instruments are summarized in Table 1 and also the extragalactic background light (EBL) corrected spectra are given in ref. (Ahnen et al. 2017).

In a recent paper Sahu et al, have explained the VHE flare of May 1 well using the photohadronic scenario by taking into account the 
Table 1. Summary of the $\gamma$-ray spectra measured by different instruments in different time intervals. The time is shown in Modified Julian Date (MJD).

\begin{tabular}{lcc}
\hline Instrument & Flux state & Period (in MJD unit) \\
\hline Whipple & low & $54936-54951$ \\
VERITAS & low & $54907-55004$ \\
MAGIC & low & $54913-55038$ \\
Whipple & high & $54952.41-54955$ \\
VERITAS & high & $54952.41-54955$ \\
MAGIC & high & 54973 \\
Whipple & very high & $54952.35-54952.41$ \\
\hline
\end{tabular}

EBL correction to the spectrum (Sahu et al. 2017b; Sahu 2019). However, the SSC spectrum used to explain the flare data was non-simultaneous with the VHE spectrum. Apart from this, the comparison between the high energy spectrum of refs. (Aliu et al. 2016) and (Ahnen et al. 2017) shows that there is a difference in their spectral behaviour.

In this work, we use the same photohadronic model to explain the spectra from low to very high states of Mrk 501 during the 4.5 months campaign. For the first time we have shown here that, very high, high and low states VHE flaring from Mrk 501 can be explained very well and simultaneously using the photohadronic scenario.

\section{THE MODEL}

Blazars are a sub class of AGN and have non thermal spectra. Rapid variability is observed in their entire electromagnetic spectra. This implies that the observed photons originate within the highly relativistic jets oriented very close to the observers line of sight (Urry \& Padovani 1995; Acciari et al. 2011). In the $v-v F_{v}$ plane, their spectral energy distributions (SEDs) have a double peak structure. These two peaks are explained by leptonic models. In this scenario, relativistic electrons radiate synchrotron photons in the jet magnetic field giving the first peak. The Compton scattering of the high energy electrons with the self-produced synchrotron photons (synchrotron self Compton, i.e. SSC) gives rise to the second peak (Dermer \& Schlickeiser 1993; Sikora, Begelman \& Rees 1994). In this model, the emitting region is a blob with comoving radius $R_{b}^{\prime}$ (the 'implies the jet is in comoving frame and without prime are in the observer frame), moving with a bulk Lorentz factor $\Gamma$ and a Doppler factor $\mathcal{D}$ (Ghisellini et al. 1998; Krawczynski et al. 2004) and for HBLs $\Gamma \simeq \mathcal{D}$.

The photohadronic model of Sahu et al. (Sahu, Miranda \& Rajpoot 2016; Sahu, de León \& Miranda 2017a; Sahu et al. 2017b; Sahu 2019; Sahu, López Fortín \& Nagataki 2019) can explain the flaring events from HBLs very well and it relies on the standard interpretation of the leptonic model discussed above to explain both low and high energy peaks by synchrotron and SSC photons respectively as in the case of any other AGN.

A double jet structure scenario is assumed to explain the multi$\mathrm{TeV}$ emission from the HBLs. The jet which is compact and smaller in size $\left(R_{f}^{\prime}\right)$ is enclosed within the bigger cone of size $R_{b}^{\prime}\left(R_{f}^{\prime}<R_{b}^{\prime}\right)$ and both have almost the same bulk Lorentz factor $\Gamma$. As the compact jet is hidden we cannot probe directly its density $n_{\gamma, f}^{\prime}$. However, the photon density in the outer region $n_{\gamma}^{\prime}$ is known from the observed flux. The photon density in the inner jet will decrease due to its adiabatic expansion when it crosses into the outer region. To connect the inner and the outer jet regions, a scaling behaviour of their photon densities is proposed as

$\frac{n_{\gamma, f}^{\prime}\left(\epsilon_{\gamma_{1}}\right)}{n_{\gamma, f}^{\prime}\left(\epsilon_{\gamma_{2}}\right)} \simeq \frac{n_{\gamma}^{\prime}\left(\epsilon_{\gamma_{1}}\right)}{n_{\gamma}^{\prime}\left(\epsilon_{\gamma_{2}}\right)}$.

In the above equation, the right hand side is known, however, the left hand is unknown. So we can use this relation to express the unknown photon density in the inner region in terms of the photon density in the outer region. The Fermi accelerated protons in the inner jet region have a power-law spectrum given by

$\frac{d N}{d E_{p}} \propto E_{p}^{-\alpha}, \quad \alpha \geq 2$.

These protons interact with the photons in the inner jet region to produce the $\Delta$-resonance. The subsequent decay of the $\Delta$-resonance to $\gamma$-rays and neutrinos takes place by intermediate $\pi^{0}$ and $\pi^{+}$states respectively.

The resonance process $p \gamma \rightarrow \Delta$ gives the kinematical condition

$E_{\gamma} \epsilon_{\gamma} \simeq 0.032 \frac{\mathcal{D}^{2}}{(1+z)^{2}} G e V^{2}$,

where $E_{\gamma}$ is the observed VHE $\gamma$-ray energy, $\epsilon_{\gamma}$ is the the background seed photon energy and $z$ is the redshift of the HBL. In the above process, the VHE photon carries about 10 per cent of the proton energy $\left(E_{p} \simeq 10 E_{\gamma}\right)$.

The observed VHE $\gamma$-ray flux is proportional to $n_{\gamma, f}^{\prime}$ and proton flux $F_{p} \equiv E_{p}^{2} d N / d E_{p}$. So the observed VHE $\gamma$-ray flux is $F_{\gamma} \propto n_{\gamma, f}^{\prime} F_{p}$. In a traditional jet scenario, the photohadronic process is inefficient due to the low photon density and superEddington luminosity (Cao \& Wang 2014; Zdziarski \& Böttcher 2015 ) in proton is needed to explain the multi-TeV emission. Using the scaling condition of equation (1), the photon density in the inner region can be expressed in terms of the observed flux (Sahu et al. 2017b; Sahu 2019).

The observed range of multi- $\mathrm{TeV} E_{\gamma}$ corresponds to the range of $\epsilon_{\gamma}$ which lies in the low energy tail region of the SSC spectrum (the beginning of the SSC spectrum). Using the relation

$n_{\gamma}^{\prime}\left(\epsilon_{\gamma}\right)=\eta\left(\frac{d_{L}}{R_{b}^{\prime}}\right)^{2} \frac{1}{(1+z)} \frac{\Phi_{S S C}\left(\epsilon_{\gamma}\right)}{\mathcal{D}^{2+\kappa} \epsilon_{\gamma}}$,

where $\eta$ is the efficiency of the SSC process and we take $\eta=1$ for 100 per cent efficiency. The parameter $\kappa=0(1)$ corresponds to continuous (discrete) blazar jet and $d_{L} \simeq 156 \mathrm{Mpc}$ is the luminosity distance of Mrk 501. So $F_{\gamma} \propto n_{\gamma}^{\prime}$ implies $F_{\gamma} \propto \Phi_{S S C} \epsilon_{\gamma}^{-1}$ and $F_{\gamma} \propto F_{p}$ implies $F_{\gamma} \propto E_{\gamma}^{-\alpha+2}$. Also remember that, high energy gamma-rays get attenuated on their way to Earth. So the observed multi-TeV spectrum has to be corrected for the EBL absorption. Taking the EBL correction into account and using equation (3), the observed multi-TeV flux can be expressed as

$F\left(E_{\gamma}\right)=A_{\gamma} \Phi_{S S C}\left(\epsilon_{\gamma}\right)\left(\frac{E_{\gamma}}{T e V}\right)^{-\alpha+3} e^{-\tau_{\gamma \gamma}\left(E_{\gamma}, z\right)}$,

where $A_{\gamma}$ is the normalization constant which can be fixed from the observed VHE data and $\tau_{\gamma \gamma}$ is the energy and redshift dependent optical depth for the pair creation process. The low energy tail region of the SSC spectrum can be expressed as $\Phi_{S S C} \propto \epsilon_{\gamma}^{\beta}$ with $\beta>0$, irrespective of whether it is in quiescent state or in flaring state. However, the value of $\beta$ does differ in different states. Using equation (3), we can express

$\Phi_{S S C}\left(\epsilon_{\gamma}\right)=\Phi_{0}\left(\frac{E_{\gamma}}{T e V}\right)^{-\beta}$. 
Substituting equation (3) in equation (5), the observed flux can be expressed as

$F_{\gamma}\left(E_{\gamma}\right)=F_{\gamma, i n}\left(E_{\gamma}\right) e^{-\tau_{\gamma \gamma}\left(E_{\gamma}, z\right)}$,

where the intrinsic flux is defined as

$F_{\gamma, i n}\left(E_{\gamma}\right)=F_{0}\left(\frac{E_{\gamma}}{T e V}\right)^{-\alpha-\beta+3}$.

The value of $\beta$ is obtained from the low energy tail region of the SSC SED of a given leptonic model. So the only free parameter here is the proton spectral index $\alpha$.

During the VHE $\gamma$-ray emission state the flux from the jet can be as high as $F_{E d d} / 2$, where $F_{E d d}$ is the Eddington flux. Also the highest energy protons emitted in the emission process should satisfy $F_{p}<$ $F_{E d d} / 2$ which will constrain the optical depth $\tau_{p \gamma}$ for the $p \gamma \rightarrow \Delta$ process and consequently the $n_{\gamma, f}^{\prime}$ in the inner region. The hidden jet lies between $R_{s}$ (Schwarzschild radius) and $R_{b}^{\prime}$ so that photon density in the inner region is high (Sahu et al. 2017b; Sahu 2019).

\section{RESULTS}

As a part of multi-wavelength campaign, an extensive study of the HBL Mrk 501 was conducted over 4.5 months prior in 2009, with the participation of Whipple 10m, VERITAS, MAGIC, and many other instruments (Ahnen et al. 2017). Although relatively large variability was measured in the VHE $\gamma$-ray and X-ray bands, overall no significant correlation was found between these energy bands. During the 4.5 months campaign period, the VHE spectra were measured in three different states: low, high and very high emission states.

Here, we shall analyse these three different states of Markarian 501 which are given in Table 1 by using the photohadronic scenario discussed above. The EBL model of Franceschini et al. (Franceschini, Rodighiero \& Vaccari 2008) is used for the EBL correction. It is observed that the EBL model of Domínguez also gives similar results (Domínguez et al. 2013). In the photohadronic model, the best fits to the VHE observed spectra are obtained using a standard optimization of parameters with the weighted least squares (WLS) method,

$W L S=\sum_{i=1}^{N} w_{i}\left(y_{i}-y^{\prime}\left(F_{0}, \alpha+\beta\right)\right)^{2}$

where $y^{\prime}$ is the predicted value of the fit with the parameters $F_{0}$ and $\alpha+\beta, y_{i}$ is the observed value, and $w_{i}$ is the weighting factor for the $i$ th value. To evaluate the goodness of the fit we used a reduced $\chi^{2}$-test. Here we neglect the systematic uncertainty of the EBL and use only the statistical uncertainty of the VHE observed flux to estimate the error of the fit.

\subsection{Low state}

The low states of Whipple, VERITAS and MAGIC are discussed here. During mid-March to early-August 2009, the average MWL SED is taken when Mrk 501 was mostly in a low state (Baring, Böttcher \& Summerlin 2017). However, for the calculation of these low states, the flaring events during the observation period are excluded from the data. In the photohadronic scenario, we need to know the tail region of the SSC spectrum which will be used to fit the VHE spectrum and to calculate the spectral index $\alpha$. The SSC tail region in the leptonic model (Baring et al. 2017) can be fitted with a power-law with $\Phi_{0}=(1.60 \pm 0.01) \times 10^{-12} \mathrm{erg} \mathrm{cm}^{-2} \mathrm{~s}^{-1}$

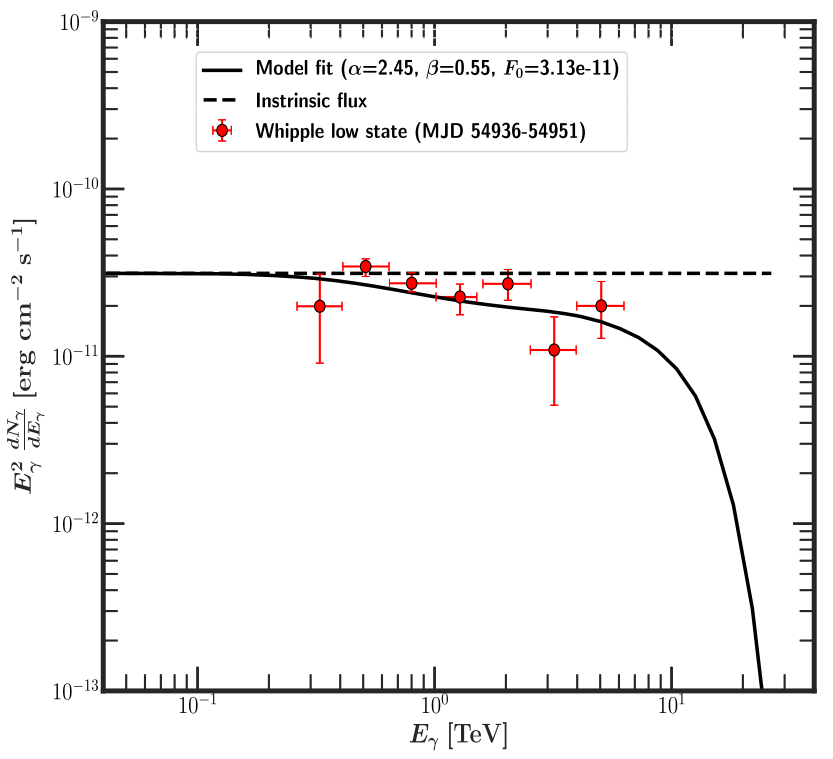

Figure 1. Whipple low state spectrum (MJD 54936 to 54951) is fitted with the photohadronic model. In all the figures the normalization constant $F_{0}$ is given in units of $\mathrm{erg} \mathrm{cm}^{-2} \mathrm{~s}^{-1}$, and the dashed curve corresponds to intrinsic flux.

and $\beta=0.55 \pm 0.01$. These values of $\Phi_{0}$ and $\beta$ are used to fit the low state spectra of Whipple, VERITAS and MAGIC and $\Gamma=15$ is used here. In the low state, the uncertainty in the fitting parameters is calculated within 10 percent of the upper/lower bounds of the observed data for Whipple, VERITAS and MAGIC. Flux below 1 $\mathrm{TeV}$ is given a weighting factor $w_{i}=0.75$, and above $1 \mathrm{TeV} w_{i}=1.0$.

\subsubsection{Whipple}

The Whipple low state spectrum between the period MJD 54936 to 54951 is observed in the energy range $0.33 \mathrm{TeV} \leq E_{\gamma} \leq$ $5.1 \mathrm{TeV}$ corresponding to the seed photon energy in the range $1.3 \mathrm{MeV} \geq \epsilon_{\gamma} \geq 20.5 \mathrm{MeV}$, which is the low energy tail region of the SSC spectrum. The low state spectrum is fitted very well with the following parameters $\alpha=(2.45)_{-0.03}^{+0.01}$ and $F_{0}=(3.13)_{-0.07}^{+0.20} \times$ $10^{-11} \mathrm{erg} \mathrm{cm}^{-2} \mathrm{~s}^{-1}$, for which we obtain $\chi_{v}^{2}=1.0$. The fitted observed spectrum (continuous curve) and the intrinsic spectrum (dashed curve) are shown in Fig. 1.

\subsubsection{VERITAS}

The low state was observed between MJD 54907 and MJD 55004 by VERITAS telescopes in the energy range $0.26 \mathrm{TeV} \leq$ $E_{\gamma} \leq 4.0 \mathrm{TeV}$ which corresponds to the SSC photon energy $1.7 \mathrm{MeV} \geq \epsilon_{\gamma} \geq 26.3 \mathrm{MeV}$. A very good fit to the data is obtained for a similar $\alpha=2.45_{-0.01}^{+0.06}$ as Whipple but $F_{0}=\left(2.56_{-0.07}^{+0.05}\right) \times$ $10^{-11} \mathrm{erg} \mathrm{cm}^{-2} \mathrm{~s}^{-1}$, for which we obtain $\chi_{v}^{2}=1.09$. The observed spectrum, fitted curve and the intrinsic spectrum are shown in Fig. 2.

\subsubsection{MAGIC}

The time averaged low state spectrum taken during MJD 54913 to MJD 55038 is in the energy range $0.14 \mathrm{TeV} \leq E_{\gamma} \leq 3.0 \mathrm{TeV}$. To produce $\Delta$-resonance this corresponds to the seed photon energy in the range $2.2 \mathrm{MeV} \leq \epsilon_{\gamma} \leq 47.7 \mathrm{MeV}$. Again a very good fit 


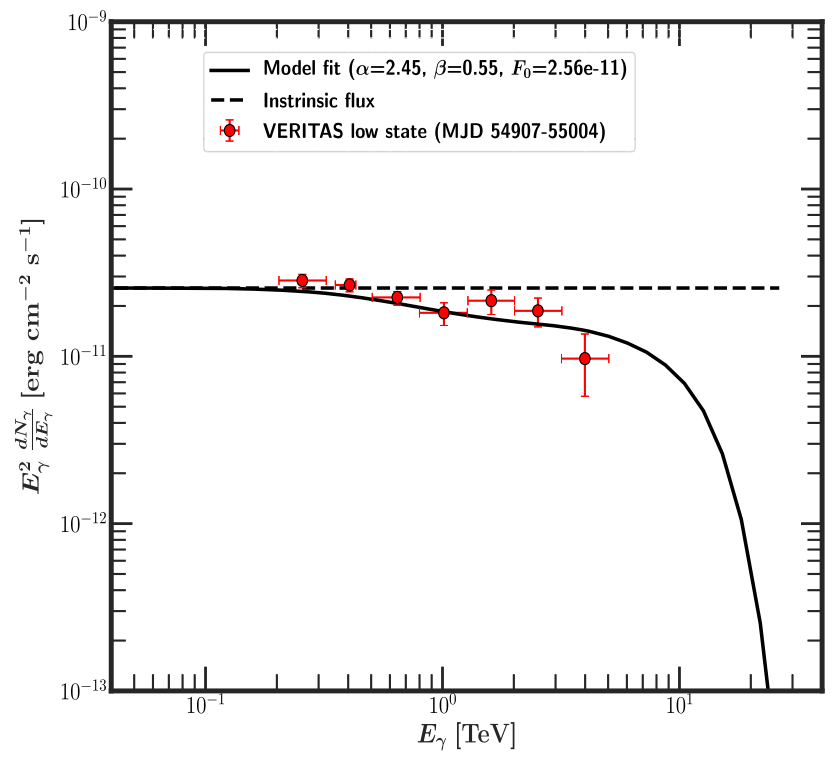

Figure 2. VERITAS low state spectrum (MJD 54907 to MJD 55004) is fitted with the photohadronic model. The corresponding intrinsic flux is also given.

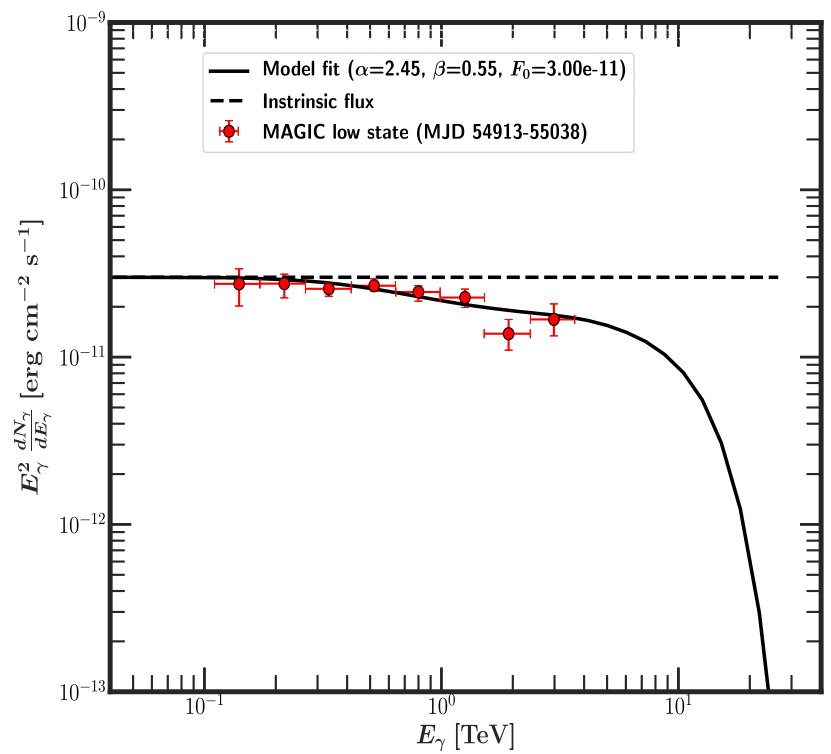

Figure 3. MAGIC low state spectrum (MJD 54913 to MJD 55038) is fitted with the photohadronic model. The corresponding intrinsic flux is also shown.

to the low state spectrum is obtained with $\alpha=(2.45)_{-0.03}^{+0.03}$ and the normalization constant is $F_{0}=\left(3.00_{-0.02}^{+0.09}\right) \times 10^{-11} \mathrm{erg} \mathrm{cm}^{-2} \mathrm{~s}^{-1}$, for which we obtain $\chi_{v}^{2}=0.99$. We also show the observed spectrum, the fitted curve and the intrinsic spectrum in Fig. 3.

As shown above the low states follow the same power-law $(\alpha=$ 2.45) except that their normalizations are different (i.e. different values of $F_{0}$ ), and their intrinsic spectra are flat irrespective of the emission period.

\subsection{High state}

The high states observed by these three telescopes are discussed below. In the high state, the uncertainty in the fitting parameters is

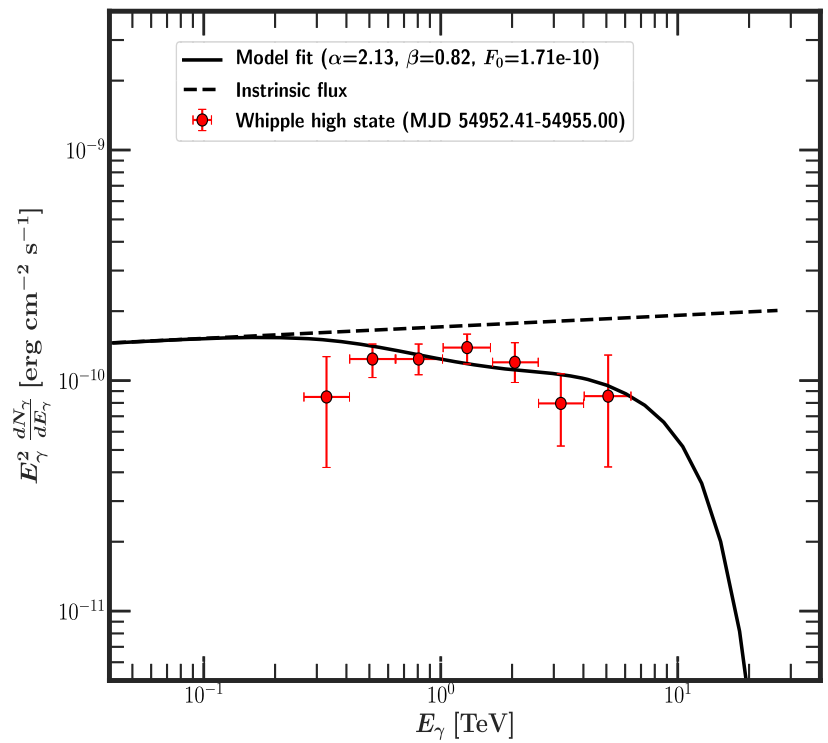

Figure 4. The Whipple high state spectrum during MJD 54952.41 to MJD 54955.00 is fitted with the photohadronic model. The corresponding intrinsic flux is also shown.

calculated within the upper/lower bounds of the observed data. Flux below $1 \mathrm{TeV}$ is given a weighting factor $w_{i}=0.85$, and above 1 $\mathrm{TeV} w_{i}=1.0$.

\subsubsection{Whipple}

The Whipple high state between MJD 54952.41 and MJD 54955.00 was observed, when the flux was about two times the base line flux. It was observed in the energy range $0.33 \mathrm{TeV} \leq E_{\gamma} \leq 5.1 \mathrm{TeV}$. The average multiwavelength SED during this period is fitted using one-zone leptonic model in ref. (Aliu et al. 2016). Using $\Gamma=15$ we observed that for the above range of $E_{\gamma}$, the SSC seed photon energy is in the range $1.3 \mathrm{MeV} \leq \epsilon_{\gamma} \leq 20.4 \mathrm{MeV}$. In this range of $\epsilon_{\gamma}$, the SSC spectrum is fitted with a power-law where $\Phi_{0}=(7.00 \pm$ $0.01) \times 10^{-12} \mathrm{erg} \mathrm{cm}^{-2} \mathrm{~s}^{-1}$ and $\beta=0.82 \pm 0.01$. In this case to have a good fit for the high state of Whipple, we obtain $\alpha=2.13_{-0.01}^{+0.03}$ and $F_{0}=\left(1.71_{-0.01}^{+0.04}\right) \times 10^{-10} \mathrm{erg} \mathrm{cm}^{-2} \mathrm{~s}^{-1}$, with a $\chi_{v}^{2}=1.01$. The calculated intrinsic flux is almost flat $\left(F_{\gamma, \text { in }} \propto E_{\gamma}^{0.05}\right)$. The data, the fitted curve, and the intrinsic flux are shown in Fig. 4.

\subsubsection{VERITAS}

The VERITAS high state corresponds to the same time period as that of Whipple i.e. MJD 54952.41 to MJD 54955.00. However, the VERITAS average flux is lower than the one observed by Whipple, which was observed in high state in the energy range $0.26 \mathrm{TeV} \leq E_{\gamma} \leq 4.0 \mathrm{TeV}$. Here we use the same SSC flux (i.e. $\beta=0.82 \pm 0.01)$ to fit the observed spectrum. A very good fit to the data is obtained for $\alpha=2.05_{-0.02}^{+0.04}$ and $F_{0}=\left(1.21_{-0.08}^{+0.07}\right) \times$ $10^{-10} \mathrm{erg} \mathrm{cm}^{-2} \mathrm{~s}^{-1}$, which is shown in Fig. 5. We obtain for this fit that $\chi_{v}^{2}=1.03$.

\subsubsection{MAGIC}

The MAGIC telescopes measured a high flux $(\sim 3$ times the low flux) on May 22nd (MJD 54973) in the energy range $0.14 \mathrm{TeV} \leq$ $E_{\gamma} \leq 3.0 \mathrm{TeV}$. However, the low energy SED was not observed 


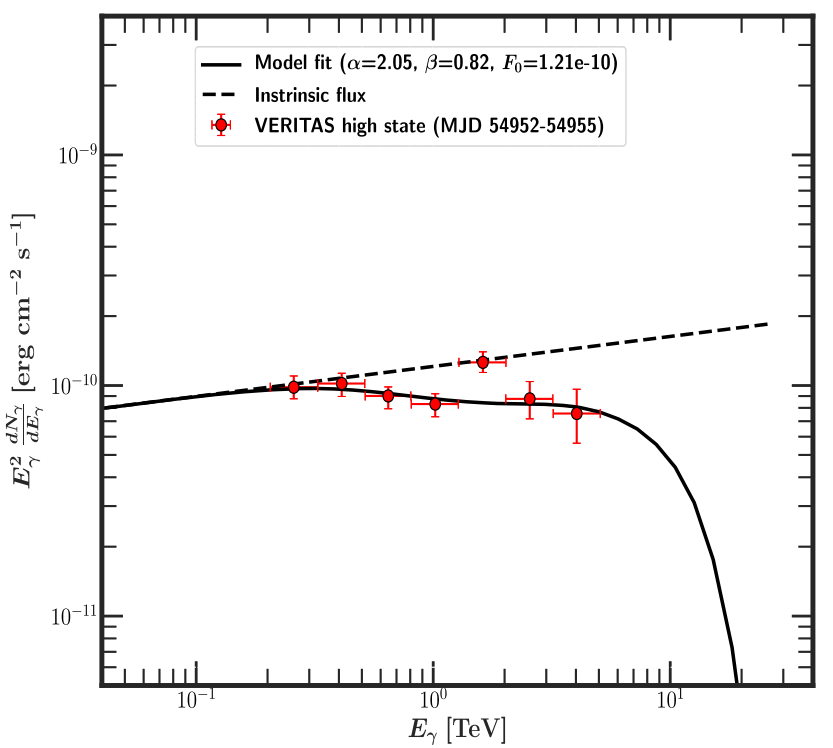

Figure 5. The VERITAS high state spectrum observed during MJD 54952.41 to MJD 54955.00 is fitted using photohadronic model and the intrinsic spectrum are shown in this figure.

strictly simultaneously during that period. As compared to the VHE flare of May 1st, this flare had a variability time scale of days, so non-simultaneous SED may not affect much in the determination of the value of $\beta$. Using the grid-scan modelling of the data obtained during the flaring episode around MJD 54973 the low energy SED is modelled in fig. 10 of ref. (Aliu et al. 2016). For the present calculation, we consider a bulk Lorentz factor $\Gamma=15$. The energy range $0.14 \mathrm{TeV} \leq E_{\gamma} \leq 3.0 \mathrm{TeV}$ implies that the seed photon energy should be in the range $47.67 \mathrm{MeV} \geq \epsilon_{\gamma} \geq 2.25 \mathrm{MeV}$ and the corresponding proton energy is in the range $1.4 \mathrm{TeV} \leq E_{p} \leq$ $30 \mathrm{TeV}$. For the above $\epsilon_{\gamma}$ range the corresponding SSC flux is a power-law with $\beta=0.63 \pm 0.01$ and $\Phi_{0}=(3.60 \pm 0.01) \times$ $10^{-12} \mathrm{erg} \mathrm{cm}^{-2} \mathrm{~s}^{-1}$. We fit the MAGIC high state spectrum with $\alpha=2.21_{-0.04}^{+0.01}$ and $F_{0}=\left(9.77_{-0.02}^{+0.18}\right) \times 10^{-11} \mathrm{erg} \mathrm{cm}^{-2} \mathrm{~s}^{-1}$, which is shown in Fig. 6, along with its intrinsic flux. For this case we obtain $\chi_{v}^{2}=1.01$.

\subsubsection{Whipple very high state}

On May 1, 2009, Whipple 10m telescope registered a very high flaring event, the flux increased by a factor $\sim 4$ in the first $0.5 \mathrm{~h}$ (MJD 54952.35-MJD 54952.37) of the observation and afterwards it decreased but remained in an elevated state during MJD 5495355 while the flux was about twice the baseline flux. The sudden rise of the flux in a short time period implies that the emission region was very small. Particularly on MJD 54952 when there was sub-hour flux variability, strictly-simultaneous observations in multiwavelength were lacking and any SED constructed during this period seems to be inconclusive. It is important to mention here that, the very high state spectrum given in fig. 8 of ref. (Aliu et al. 2016) and the recent one in fig. 4 of ref. (Ahnen et al. 2017) are different even though they were taken in the same period (MJD 54952.35-MJD 52952.41), and the former one has a lower flux. We observe that these spectra have different values of $\alpha$ for the same $\beta$.

Due to non-simultaneous observation of the SSC SED and rapid variability of the very high state, we don't know how the tail region

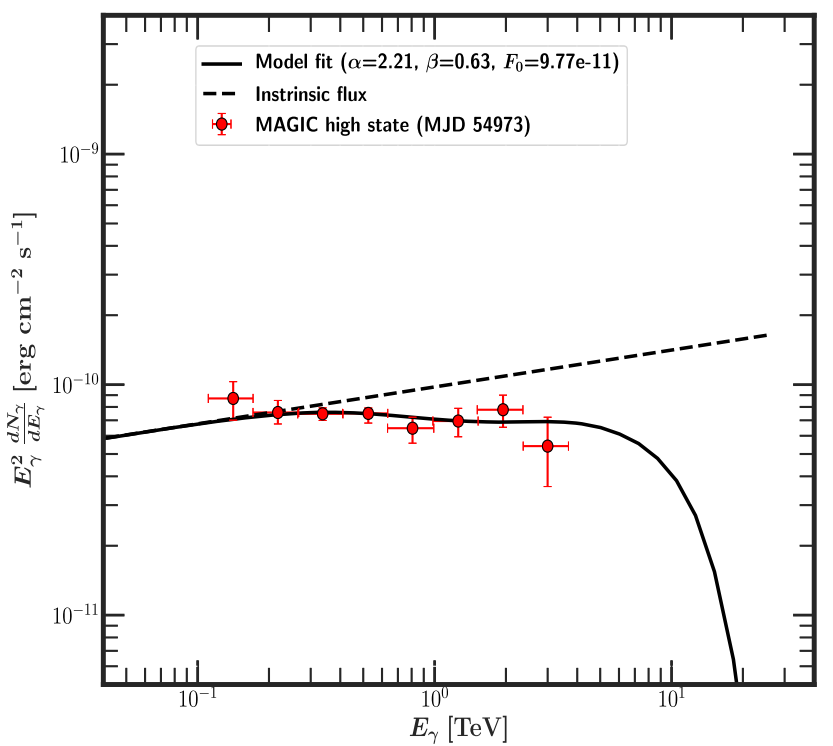

Figure 6. MAGIC high state spectrum observed on MJD 54973 is fitted with the photohadronic model. The corresponding intrinsic flux is also shown.

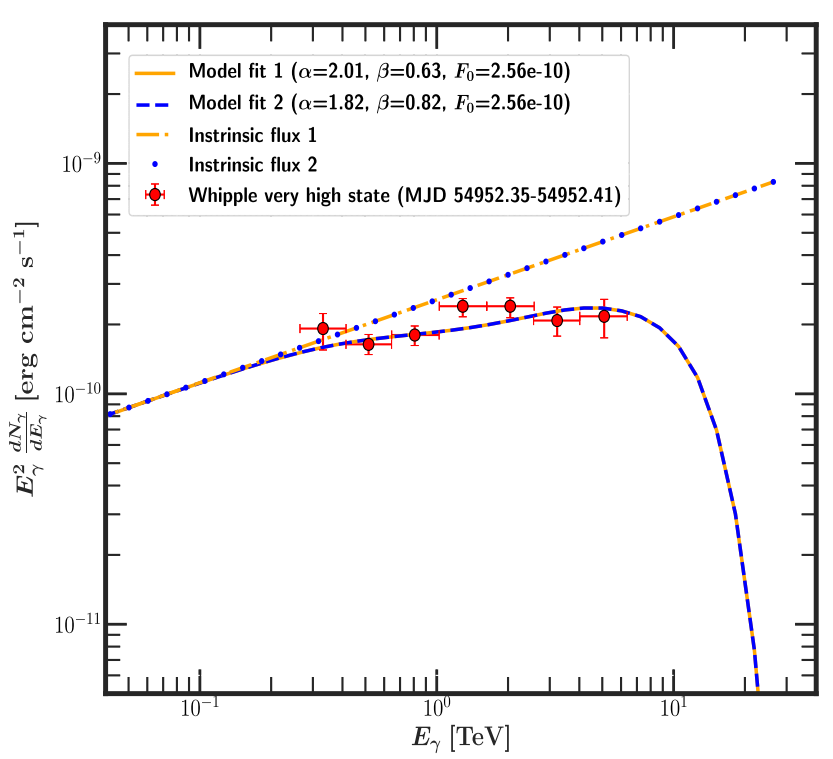

Figure 7. The Whipple very high state flaring spectrum during MJD 54952.35 to MJD 54952.37 is fitted with the photohadronic model. The corresponding intrinsic flux is also shown. For $\beta=0.63$ and $\beta=0.82$, we obtain exactly the same fit to the observed spectrum because $\alpha+\beta=2.63$ for both cases.

of the SSC flux behaves, hence the value of $\beta$ is uncertain. The bulk Lorentz factor $\Gamma$ during this rapid variability period is also unknown. However, to explain the high state emissions, $\beta=0.82 \pm 0.01$ was used for the period MJD 54952.41-54955 which was immediately after the very high state. Another high state observed on MJD 54973 is explained with $\beta=0.63 \pm 0.01$. So here we use both values of $\beta$ to fit the observed very high state spectrum. A very good fit to the spectrum is obtained for $\alpha=2.01_{-0.01}^{+0.01}, \beta=0.63$, and $F_{0}=\left(2.56_{-0.01}^{+0.08}\right) \times 10^{-10} \mathrm{erg} \mathrm{cm}^{-2} \mathrm{~s}^{-1}$, and $\alpha=1.82_{-0.01}^{+0.01}, \beta=$ $0.82 \pm 0.01$, and $F_{0}=\left(2.56_{-0.01}^{+0.08}\right) \times 10^{-10} \mathrm{erg} \mathrm{cm}^{-2} \mathrm{~s}^{-1}$, which are exactly the same as shown in Fig. 7. Flux below $1 \mathrm{TeV}$ is 


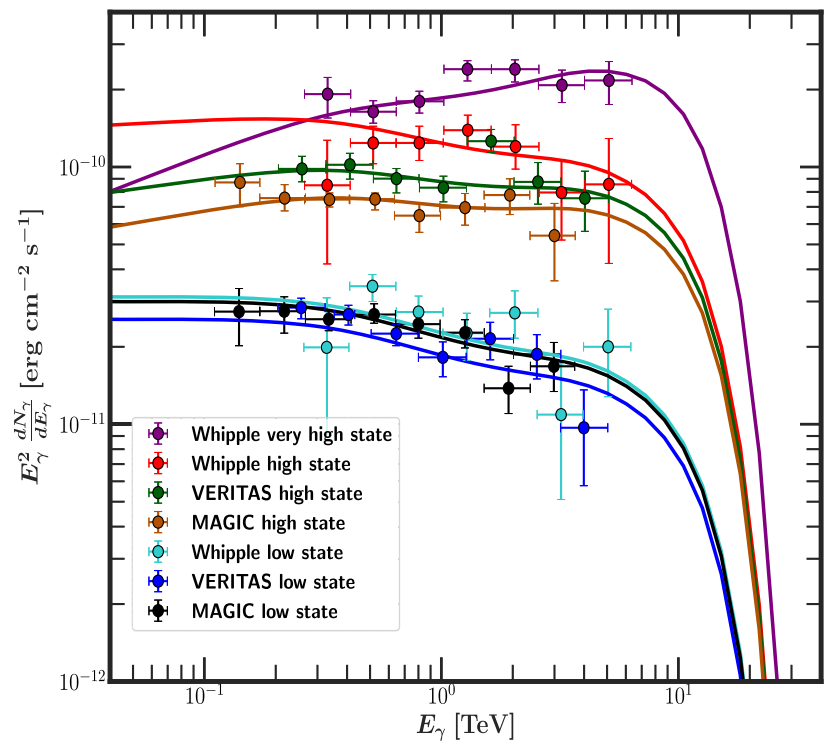

Figure 8. Photohadronic fit to the low, high and very high emission states are shown together along with the observed data for comparison.

assigned a weighting factor $w_{i}=0.75$, and above $1 \mathrm{TeV} w_{i}=$ 1.0 , and for both fits we obtain $\chi_{v}^{2}=0.99$. As we know, the proton spectral index $\alpha$ should be $\geq 2$. Hence, we only consider the first case for which the intrinsic flux $F_{\gamma, \text { in }} \propto E_{\gamma}^{0.37}$. It seems, the SSC SED during the very high state emission must have $\beta \leq 0.63$. For comparison we show the spectra of the low, the high, and the very high emission states observed by different instruments and their respective photohadronic fits in Fig. 8.

In the flaring state, as has been alluded to before, in general the flux of the two opposing jets can be as high as $F_{E d d} / 2$. Mrk 501 has a central black hole of mass $M_{B H}=(0.9-3.5) \times 10^{9} M_{\odot}$ which corresponds to an Eddington luminosity $L_{E d d}=(1.13-4.4) \times$ $10^{47} \mathrm{erg} \mathrm{s}^{-1}$ and $F_{E d d}=(3.9-15.0) \times 10^{-8} \mathrm{erg} \mathrm{cm}^{-2} \mathrm{~s}^{-1}$. The proton flux $F_{p}$ corresponding to the highest $\gamma$-ray of $E_{\gamma}=5.09$ TeV must satisfy $F_{p}<F_{E d d} / 2=2.0 \times 10^{-8} \mathrm{erg} \mathrm{cm}^{-2} \mathrm{~s}^{-1}$. Using the relation between the proton flux and the $\gamma$-ray flux we obtain $\tau_{p \gamma}$ $>0.08$. For a moderate efficiency of the $p \gamma$ process we take $\tau_{p \gamma}=$ 0.1 for which we obtain $F_{p}=1.6 \times 10^{-8} \mathrm{erg} \mathrm{cm}^{-2} \mathrm{~s}^{-1}<F_{E d d} / 2$, which shows that the highest energy proton has sub-Eddington luminosity.

It is very important to note that during different VHE emission states from Mrk 501, the multiwavelength SEDs are different in the SSC frequency range (different values of $\beta$ ) which is obvious from the leptonic model fit to the SEDs. Or in other words, this corresponds to different seed photon densities in the SSC band in each epoch of flaring as given in equation (4). But the proton spectral index lies in a narrow range $2.00 \lesssim \alpha \lesssim 2.45$ which shows that the high energy proton acceleration mechanism is the same for low, high and very high flaring states. We have observed that the photohadronic model works well for $E_{\gamma} \gtrsim 100 \mathrm{GeV}$ and in this energy range the SSC contribution is negligible.

\section{CONCLUSIONS}

The HBL Mrk 501 was observed during a multiwavelength campaign covering a period of 4.5 months from March 15 to August 1, 2009 (Ahnen et al. 2017). In this period three different types of
VHE emissions were observed by Whipple 10m, VERITAS and MAGIC telescopes. A very high state flaring event was observed only by Whipple telescope for about $0.5 \mathrm{~h}$ when the flux had a dramatic increase. All the three telescopes also observed high emission state and low emission state of Mrk 501 during this campaign period. Using the photohadronic scenario, where Fermi accelerated protons interacting with the seed photons in tail region of the SSC SED in the inner jet region produce the $\Delta$-resonance and its decays to neutral pion will subsequently produce observed VHE photons. We have shown that all these three types of VHE spectra can be fitted very well with the photohadronic scenario when absorption by EBL is accounted for. Also we observed that the intrinsic spectra of these three different states are different from each other. The low state spectrum is almost flat which shows that $F_{\gamma, \text { in }}$ is constant and the one from the high state is $\propto E_{\gamma}^{0.1}$. The $F_{\gamma, \text { in }}$ from the very high state is found to be proportional to $E_{\gamma}^{0.37}$. So going from low state to very high state, the intrinsic flux slowly increases from a constant to a power-law, and simultaneously the observed flux follows the trend from downward going to flat to upward going which is clearly seen in Fig. 8. We also observed that the proton spectral index for all these cases lies in a small window of $2.00 \lesssim \alpha \lesssim 2.45$. It seems that for low, high, and very high state emissions from Mrk 501, the acceleration mechanism for the high energy proton is the same, and the only difference is coming from the seed photon density (different values for $\beta$ ). It is important that we should have simultaneous multiwavelength observations of the flaring event to constrain the photohadronic model.

\section{ACKNOWLEDGEMENTS}

The work of SS is partially supported by DGAPA-UNAM (Mexico) Project No. IN103019. SN is partially supported by 'JSPS Grantsin-Aid for Scientific Research <KAKENHI> (A) 19H00693', 'Pioneering Program of RIKEN for Evolution of Matter in the Universe (r-EMU)', and 'Interdisciplinary Theoretical and Mathematical Sciences Program of RIKEN'. SS is also thankful to Japan Society for the Promotion of Science (JSPS) for the invitational fellow program.

\section{REFERENCES}

Abdo A. A. et al., 2009, Astrophys. J., 699, 31

Acciari V. A. et al., 2011, Astrophys. J., 729, 2

Aharonian F. A., 1999, Astron. Astrophys., 349, 11

Aharonian F. A., 2001, Astron. Astrophys., 366, 62

Aharonian F. A. et al., 1999, Astron. Astrophys., 342, 69

Ahnen M. L. et al., 2017, Astron. Astrophys., 603, A31

Aliu E. et al., 2016, Astron. Astrophys., 594, A76

Baring M. G., Böttcher M., Summerlin E. J., 2017, Mon. Not. Roy. Astron. Soc., 464, 4875

Cao G., Wang J., 2014, Astrophys. J., 783, 108

Dermer C. D., Schlickeiser R., 1993, Astrophys. J., 416, 458

Domínguez A.., Finke J. D., Prada F., Primack J. R., Kitaura F. S., Siana B., Paneque D., 2013, Astrophys. J., 770, 77

Franceschini A., Rodighiero G., Vaccari M., 2008, Astron. Astrophys., 487, 837

Ghisellini G., Celotti A., Fossati G., Maraschi L., Comastri A., 1998, Mon. Not. Roy. Astron. Soc., 301, 451

Gliozzi M., Sambruna R. M., Jung I., Krawczynski H., Horan D., Tavecchio F.., 2006, Astrophys. J., 646, 61

Katarzyński K., Sol H., Kus A., 2001, Astron. Astrophys., 367, 809

Krawczynski H. et al., 2004, Astrophys. J., 601, 151 
Pian E. et al., 1999, ASP Conf. Ser., 159, 180

Sahu S., 2019, Rev. Mex. Fis., 65, 307

Sahu S., Miranda L. S., Rajpoot S., 2016, Eur. Phys. J., C76, 127

Sahu S., de León A. R., Miranda L. S., 2017a, Eur. Phys. J., C77, 741

Sahu S., Yáñez M. V. L., Miranda L. S., de León A. R., Gupta V., 2017b, Eur. Phys. J., C77, 18

Sahu S., López Fortín C. E., Nagataki S., 2019, Astrophys. J. L., 884, L17

Sambruna R. M. et al., 2000, ApJ, 538, 127
Sikora M., Begelman M. C., Rees M. J., 1994, Astrophys. J., 421, 153

Tavecchio F. et al., 2001, Astrophys. J., 554, 725

Urry C. M., Padovani P., 1995, Publ. Astron. Soc. Pac., 107, 803

Villata M., Raiteri C. M., 1999, Astron. Astrophys., 347, 30

Zdziarski A. A., Böttcher M., 2015, Mon. Not. Roy. Astron. Soc., 450, L21

This paper has been typeset from a $\mathrm{T}_{\mathrm{E}} \mathrm{X} / \mathrm{LT} \mathrm{E} \mathrm{X}$ file prepared by the author. 